\title{
Discovery of the first potent and selective Mycobacterium tuberculosis Zmp1 inhibitor
}

\author{
Mattia Mori ${ }^{\mathrm{a}, \mathrm{b}}$, Francesca Moraca ${ }^{\mathrm{a}}$, Davide Deodato ${ }^{\mathrm{a}}$, Davide M. Ferraris ${ }^{\mathrm{c}}$, Petra Selchow ${ }^{\mathrm{d}}$, \\ Peter Sander ${ }^{\mathrm{d}, \mathrm{e}}$, Menico Rizzi ${ }^{\mathrm{c}, *}$, Maurizio Botta ${ }^{\mathrm{a}, \mathrm{f}, *}$ \\ ${ }^{a}$ Dipartimento di Biotecnologie, Chimica e Farmacia, University of Siena, Via Aldo Moro 2, 53100 Siena, Italy \\ ${ }^{\text {b } C L N S ~ I s t i t u t o ~ I t a l i a n o ~ d i ~ T e c n o l o g i a @ S a p i e n z a, ~ V i a l e ~ R e g i n a ~ E l e n a ~ 291, ~} 00161$ Roma, Italy \\ ' Dipartimento di Scienze del Farmaco, University of Piemonte Orientale 'A. Avogadro', Viale Largo Donegani 2, 28100 Novara, Italy \\ ${ }^{\mathrm{d}}$ Institute of Medical Microbiology, University of Zurich, Gloriastrasse 32, CH-8006 Zurich, Switzerland \\ e National Reference Laboratory for Mycobacteria (NRLM), Gloriastrasse 32, CH-8006 Zurich, Switzerland \\ f Sbarro Institute for Cancer Research and Molecular Medicine, Center for Biotechnology, College of Science and Technology, Temple University, \\ BioLife Science Bldg., Suite 333, 1900 N 12th Street, Philadelphia, PA 19122, USA
}

\section{A R T I C L E I N F O}

\section{Article history:}

Received 18 March 2014

Revised 31 March 2014

Accepted 2 April 2014

Available online 13 April 2014

\section{Keywords:}

Tuberculosis

Zmp1

Virtual screening

Enzyme inhibitors

Metalloproteases

\begin{abstract}
A B S T R A C T
The Mycobacterium tuberculosis extracellular zinc metalloprotease 1 (Zmp1) has been proposed to play a key role in phagosome maturation and to enhance the survival of Mycobacterium tuberculosis in the host. Consequently, small molecule inhibitors of Zmp1 are of pivotal importance as a tool to better understand the pathogenicity of Zmp1 and as lead candidates for pharmacological intervention. Here we combined in silico structure-based inhibitor design with biochemical studies to discover and characterize the first potent competitive Zmp1 inhibitor showing a $K_{\mathrm{i}}$ of $94 \mathrm{nM}$ and a high selectivity for Zmp1 with respect to human Neprilysin.
\end{abstract}

(C) 2014 Elsevier Ltd. All rights reserved.
It is widely appreciated that novel pharmacological approaches against tuberculosis (TB) are urgently needed and may be stimulated by the characterization and validation of novel protein targets that sustain tuberculosis pathogenicity. In this respect, the Mycobacterium tuberculosis (Mtb) zinc-dependent metalloprotease 1 (Zmp1), a member of the M13 endopeptidase family, ${ }^{1,2}$ has been proposed to play a key role during phagosome maturation and, therefore, can be proposed as a potential therapeutic target against TB.

Following host infection, Mtb is phagocytized by macrophages into the phagosome that, in turn, should undergo to a critical maturation step which culminates with the formation of the phagolysosome that leads to Mtb clearance. ${ }^{3}$ However, Mtb has been found to inhibit the phagosome maturation step, precluding macrophage killing and remaining indefinitely in a latent non-virulent form in infected persons. Mtb is then re-activated as soon as an immunosuppressive condition occurs, thus preventing a definitive eradication of the pathogen from the host, giving rise to TB recurrences. ${ }^{4}$

\footnotetext{
* Corresponding authors. Tel.: +39 0577 234306; fax: +39 0577234333 (M.B.); Tel.: +39 0321 375712; fax: +390321 375821 (M.R.).

E-mail address: botta.maurizio@gmail.com (M. Botta).
}

Zmp1 has been proposed to impact on the phagosome maturation by inhibiting caspase- 1 activation and the processing of proIL-1 $\beta$ into IL- $1 \beta .^{3}$ Indeed, the suppression of the $z m p 1$ gene has been found to reactivate caspase- 1 as well as the production of IL-1 $\beta$, thus leading to phagosome maturation and the clearance of the pathogen. ${ }^{5}$ A recent report by Muttucumaru et al. claims that deletion of the zmp1 gene causes bacterial hypervirulence in a murine model, ${ }^{6}$ contrarily to what was previously observed by Master and colleagues, where zmp1 deletion led to virulence attenuation. ${ }^{3}$ Therefore, the precise biological role of $\mathrm{Zmp} 1$ in either Mtb virulence or host immune response escape is still under debate and has been not fully deciphered yet, although it is clear that the enzyme plays a major role in the host-pathogen interaction. Indeed, a BCG mutant in which the zmp1 gene has been deleted (zmp1 rBCG) was developed ${ }^{7}$ and preclinical studies of the $\mathrm{rBCG}$ $\Delta z \mathrm{mp} 1$ vaccine are currently in progress. ${ }^{8}$ Therefore, the discovery of Zmp1 inhibitors is of high relevance to better assessing the role played by the enzyme in the pathogenicity of tuberculosis. Moreover, the fact that Zmp1 localizes outside the Mtb cell wall, which is difficult to penetrate, reinforces $\mathrm{Zmp} 1$ as attractive pharmacological target for the development of small molecule inhibitors. Recently, the first crystal structure of $\mathrm{Zmp} 1$ in complex with the 
generic zinc metalloprotease inhibitor phosphoramidon has been solved at $2.60 \AA$ resolution (PDB ID: $3 Z \mathrm{ZUK}){ }^{9}$ elucidating key Zmp1/inhibitor interactions and representing therefore a valuable tool to design specific small molecule Zmp1 inhibitors by means of computer-aided drug design.

In this multidisciplinary work, we combined a two-round structure-based virtual screening with organic synthesis, enzymatic and kinetics assays to discover potent Zmp1 inhibitors endowed with a significant chemical diversity with respect to generic metalloprotease inhibitor phosphoramidon. Starting from the crystallographic structure of $\mathrm{Zmp} 1 /$ phosphoramidon complex, ${ }^{9}$ the chain A was retained due to the less number of gaps with respect to the chain B. Preliminary self-docking trials were performed by using different docking programs (GOLD, Glide, AutoDock) ${ }^{10,11}$ to probe their capability to reproduce the crystallographic binding mode of phosphoramidon. Due to the general failure of the self-docking procedure, also after removal of the rhamnose moiety from the co-crystalized inhibitor, the chain A of Zmp1/phosphoramidon was submitted to energy minimization in explicit solvent by means of Amber11. ${ }^{12}$ Repeating the self-docking of phosphoramidon towards such refined Zmp1 structure provided satisfactory results. Moreover, different protonation schemes for zinc-binding residues His493, His497 and Glu560, for Glu494 and His622 (Zmp1 residues are numbered as in the abovementioned X-ray structure) were tested and the best agreement between computational and experimental pose of phosphoramidon was obtained by keeping the non-zinc-binding residues Glu494 and His622 in their protonated form (Supplementary information). The Goldscore function of GOLD (version 4.0.1) provided the lowest root mean square deviation (RMSD $=0.315 \AA$ ) between the docking-based and the energy minimized crystallographic binding pose of phosphoramidon (Supplementary information). In line with previous reports, here we obtained significant benefits by performing structure-based ligand design towards computationally refined protein structures. $^{13,14}$

After these preliminary molecular modeling analysis, we performed virtual screening by means of a protocol composed of four subsequent steps: (i) a fast screening with $\operatorname{ROCS}^{15}$ (version 3.1.2,
Openeye Scientific Software); (ii) docking of the most promising hits identified by ROCS towards Zmp1; (iii) rescoring by means of the Molecular Mechanics Generalized Born Surface Area method (MM-GBSA) and (iv) visual inspection. First, we generated a ROCS query based on the energy minimized binding mode of phosphoramidon deprived of the rhamnose moiety, because it is mostly solvent exposed and does not interact directly with $\mathrm{Zmp} 1$ residues. $^{9}$ The query was then used to screen the whole Asinex database (about 600,000 small molecules) and the $10 \%$ top ranking molecules sorted by the Tanimoto Combo score were docked towards the catalytic site of $\mathrm{Zmp} 1$ in its energy minimized structure. It is widely appreciated that rescoring docking poses with an external potential energy function generally improve the quality of a virtual screening protocol. Based on our experience, the ligand delta energy of binding of the 1000 top ranking small molecules was then calculated by means of the MM-GBSA method. ${ }^{14}$ Most of these molecules bear a well-known zinc binding group, mostly carboxylic acid or sulfonate groups. Finally, top ranking 500 small molecules endowed with the lowest MM-GBSA delta energy of binding to Zmp1 were visually inspected. Among them, 18 most interesting molecules were selected (ZTB1-ZTB18), based on a combination of binding mode, rescoring energy and chemical diversity each other as well as with respect to phosphoramidon or other known Zn-proteases inhibitors, and submitted to biochemical investigations. Chemical structures of selected compounds, the detailed experimental procedure adopted for carrying out the enzymatic assays on recombinant Mtb Zmp1 and human Neprilysin, and for the calculation of the $K_{\mathrm{i}}$ values for the different inhibitors are reported as Supplementary information.

Four out of the eighteen molecules (Fig. 1) proved to inhibit Zmp1 catalytic activity at $100 \mu \mathrm{M}$, with ZTB12 being the most active with a $K_{\mathrm{i}}$ of $32.9 \pm 0.4 \mu \mathrm{M}$ (Table 1$)$. The docking-based binding mode of ZTB12 (Supplementary information) shows the sulfonate group interacting with Glu494 and His622 as well as with the catalytic $\mathrm{Zn}(\mathrm{II})$ in a geometry that resembles zinc coordination. The aromatic core is stacked with the side chain of Arg616, whereas the non-zinc-binding sulfonate establishes H-bonds with the guanidine group of Arg616.
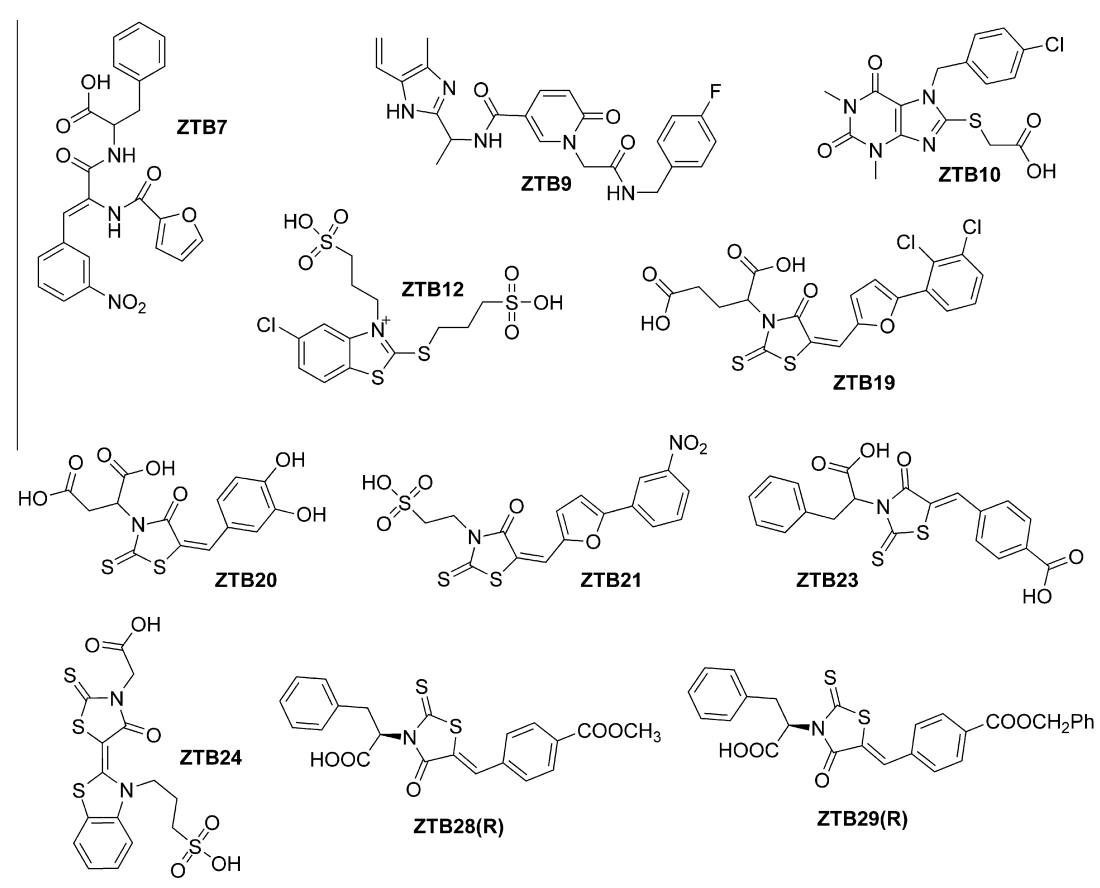

Figure 1. Chemical structure of active $\mathrm{Zmp} 1$ inhibitors described in this work. 
Table 1

Inhibition of Zmp1 catalytic activity by small molecules selected in the first-round virtual screening

\begin{tabular}{lll}
\hline Compd $^{\mathrm{a}}$ & \% Inhibition at $100 \mu \mathrm{M}$ & $K_{\mathrm{i}}(\mu \mathrm{M})$ \\
\hline ZTB7 & 54 & n.d \\
ZTB9 & 16 & n.d \\
ZTB10 & 28 & n.d \\
ZTB12 & 78 & $32.9 \pm 0.4$
\end{tabular}

a Molecules not showing inhibition at tested conditions are not reported in the table.

b Not determined.

Since ZTB12 was the most potent hit identified in the firstround virtual screening, it was selected as the reference starting point of the second-round virtual screening, which aimed at improving the ligand inhibitory potency towards Zmp1. A new ROCS query was built on the docking-based binding conformation of ZTB12 and used to screen the 'Drugs Now' subset of the ZINC database, which was composed of over six million compounds (the docking conformation of ZTB12 and the respective ROCS query are in Supplementary information). 10\% top-ranking molecules filtered by ROCS were selected for docking. However, due to the large dimension of such test-set, only compounds bearing at least a carboxyl or a sulfonate zinc binding groups were docked towards Zmp1 (about 11,000 molecules). After rescoring and visual inspection, nine molecules (ZTB19-ZTB27, see Supplementary information) were deemed top priority and submitted to in vitro tests using pure recombinant Mtb Zmp1. Results of enzymatic assays showed that five out of the nine compounds delivered by the second-round virtual screening proved to inhibit Zmp1 in vitro with higher potency than ZTB12 (Table 2 and Fig. 1).

A significant improvement of the hit enrichment was observed, as more than $55 \%$ of molecules selected in silico were active in vitro. The most potent Zmp1 inhibitor was ZTB23 with a $K_{\mathrm{i}}$ of $0.39 \pm 0.088 \mu \mathrm{M}$. Since ZTB23 was purchased as racemic mixture of $-\mathrm{R}$ and $-\mathrm{S}$ enantiomers (thereafter referred as $\mathbf{Z T B 2 3}(\mathbf{R})$ and ZTB23(S), respectively), in depth docking studies were performed to predict their binding mode and affinity to $\mathrm{Zmp} 1$ as well as both enantiomers were synthesized by means of stereoselective synthesis. Molecular modeling suggested that, in both enantiomers, the carboxyl group of the phenylalanine moiety interacts with the catalytic zinc ion in a geometry resembling zinc coordination, whereas significant differences were observed for the remaining portion of the molecules. In particular, the L-phenylalanine aromatic ring of ZTB23(S) is well inserted into the hydrophobic S1 ${ }^{\prime}$ cavity with the rhodanine moiety connecting the zinc-binding portion of the inhibitor with the benzoic acid moiety, which take place in proximity of the large S2' sub-site and interacts with the nonconserved Arg616. In the docking-based binding mode of ZTB23(R) (Fig. 2), the D-phenylalanine aromatic ring is projected towards the solvent in a region occupied by the sugar moiety of phosphoramidon in its crystallographic pose, ${ }^{9}$ whereas the rhodanine performs a H-bond with Asn452 and the benzoic acid moiety is well inserted in the large S2' sub-site where it interacts with Thr606. Notably, ZTB23(R) establishes profitable H-bond interactions with Glu494 and His622, which may reinforce the inhibitor affinity to Zmp1 and may lead to a higher inhibitory potency.

Enantiomerically pure ZTB23(S) and $\mathbf{Z T B 2 3}(\mathbf{R})$ were stereoselectively synthesized as outlined in Scheme 1 (the synthesis of ZTB23(R) is reported). The rhodanine $\mathbf{3 3}$ was obtained by following the protocol described by Kumar et al., ${ }^{16}$ in which commercial optically pure phenylalanine (30) was reacted with carbon disulfide in aqueous sodium hydroxide to give 31. The latter was reacted in situ with sodium chloroacetate giving $\mathbf{3 2}$ that was lastly cyclized under acidic conditions affording compound 33 (66\%

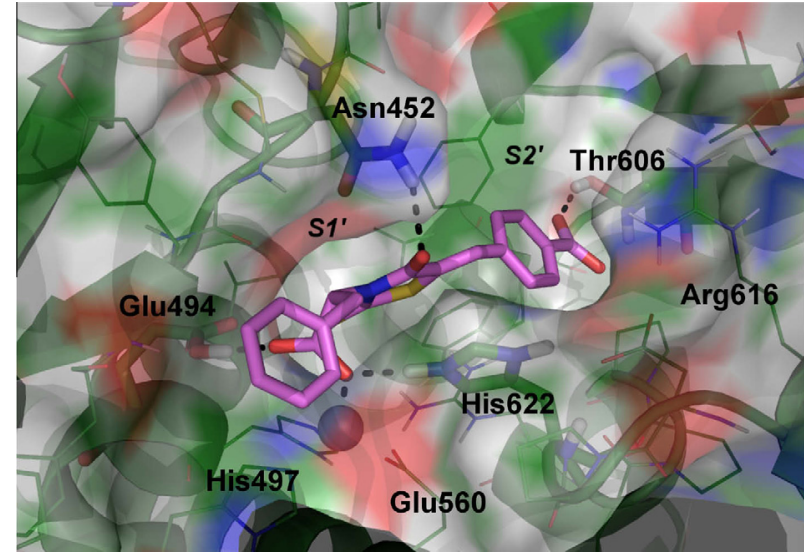

Figure 2. Docking based binding mode of $\mathbf{Z T B 2 3}(\mathbf{R})$ within the catalytic site of Zmp1. Polar contacts and zinc coordination are highlighted as black dashed lines. ZTB23(R) is showed as violet sticks. Zmp1 is showed as green cartoon and transparent surface, with key residues showed as sticks.

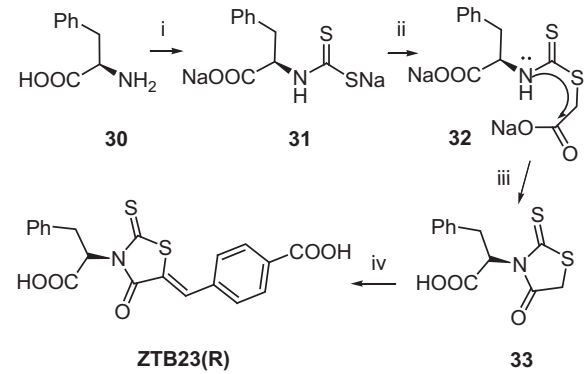

Scheme 1. Synthesis of title compound, reaction and conditions: (i) carbon disulfide, $\mathrm{NaOH}, \mathrm{H}_{2} \mathrm{O}$, rt overnight; (ii) sodium chloroacetate solution, $\mathrm{rt} 2 \mathrm{~h}$; (iii) $6 \mathrm{~N} \mathrm{HCl}$, two drops $\mathrm{POCl}_{3}, 75^{\circ} \mathrm{C}, 4 \mathrm{~h}$; (iv) p-carboxybenzaldehyde, $\beta$-alanine $\mathrm{CH}_{3} \mathrm{COOH}$ reflux, $6 \mathrm{~h}$.

isolated yield). The final Knoeveneagel condensation between $\mathbf{3 3}$ and $p$-carboxybenzaldehyde in acetic acid furnished $\mathbf{Z T B 2 3 ( R )}$ in very good isolated yield (83\%) and high enantiomeric purity as demonstrated by HPLC analysis (Supplementary information). The synthesis of ZTB23(S) follows the same synthetic pipeline starting with the L-phenylalanine (Supplementary information). Based on a survey performed on Scifinder and PubChem databases performed at the time of experiments, we may claim that this is the first report on the synthesis of active inhibitors $\mathbf{Z T B 2 3}(\mathbf{R})$ and ZTB23(S). ${ }^{17}$

Enzymatic assays showed a $K_{\mathrm{i}}$ of $1.7 \pm 0.3 \mu \mathrm{M}$ for $\mathbf{Z T B 2 3}(\mathbf{S})$ and a $K_{\mathrm{i}}$ of $0.094 \pm 0.054 \mu \mathrm{M}$ for $\mathbf{Z T B 2 3}(\mathbf{R})$ (Table 2), reinforcing the molecular modeling evidence that performing profitable $\mathrm{H}$-bonds within the Zmp1 catalytic site as well as interacting within the depth S2' pocket might be key determinants for potent inhibition of Zmp1. Notably, a significant selectivity of ZTB23(R) was observed with respect to the human Neprilysin (selectivity index $>250$, Table 2), which is the human enzyme most similar to Zmp1 according to a BLASTP search (http://blast.ncbi.nlm.nih. $\operatorname{gov} /)^{18}$

The methyl and benzyl ester derivatives of $\mathbf{Z T B 2 3}(\mathbf{R})$, namely ZTB28(R) and ZTB29(R), were also synthesized as described in Supplementary information and tested for Zmp1 inhibition. Both molecules proved to inhibit Zmp1 catalytic activity with a weaker potency than $\mathbf{Z T B 2 3}(\mathbf{R})$ (Table 2), thus highlighting the key role of the non-zinc binding benzoic acid group for Zmp1 inhibition. 
Table 2

Inhibition of Mtb Zmp1 and human Neprilysin catalytic activity by small molecules selected from the second-round virtual screening.

\begin{tabular}{llll}
\hline Compd & $K_{\mathrm{i}}(\mu \mathrm{M})$ Mtb Zmp1 & $K_{\mathrm{i}}(\mu \mathrm{M})$ human Neprilysin & SI $^{\mathrm{d}}$ \\
\hline ZTB12 $^{\mathrm{a}}$ & 32.90 & n.d. & n.d. \\
ZTB19 $_{\text {ZTB20 }}$ & 3.70 & n.d. & n.d. \\
ZTB21 & 2.70 & n.d. & n.d. \\
ZTB23 $^{\mathrm{b}}$ & $0.39 \pm 0.09$ & n.d. & n.d. \\
ZTB24 $_{\text {ZTB23 }(\mathbf{S})}$ & 4.80 & n.d. & n.d. \\
ZTB23 $(\mathbf{R})_{\text {ZTB28 }(\mathbf{R})}$ & $0.094 \pm 0.30$ & n.d. & n.d. \\
ZTB29(R) & $1.8 \pm 0.3$ & 37 & 21.7 \\
\hline
\end{tabular}

a Compound 12 has been kept for comparison.

b Racemic mixture.

c Not determined.

d Selectivity index calculated as $K_{\mathrm{i}}$ (Neprilysin)/K $K_{\mathrm{i}}(\mathrm{Mtb} \mathrm{Zmp} 1)$ ratio.

Finally, kinetics studies showed that all compounds herein discovered inhibit $\mathrm{Zmp} 1$ with a competitive mechanism with respect to the generic fluorogenic substrate for Zn-metalloproteases MMP2/MMP7 (Calbiochem Prod. Num 03-32-5032).

In conclusion, we used a multidisciplinary approach mixing molecular modeling, organic chemistry and biochemistry to discover potent inhibitors of the Zmp1 of Mtb. The most potent inhibitor is $\mathbf{Z T B 2 3 ( R )}$ showing inhibition at nanomolar concentration in vitro and a significant selectivity with respect to human Neprilysin. ZTB23(R) is therefore a useful tool to investigate the biological functions of $\mathrm{Zmp} 1$ during infection also at the light of the increasing interest that the BCG mutant-based vaccine $z m p 1 \mathrm{rBCG}$ is receiving as a potential novel vaccines against TB. ${ }^{7}$ Notably, ZTB23 $(R)$ is also a valuable lead candidate for further optimization finalized to the development of pharmaceutically relevant anti-TB candidate agents. To the best of our knowledge, this is the first medicinal chemistry study focusing on the identification of potent Zmp1 inhibitors.

\section{Acknowledgments}

This work was partially supported by the European Union FP7 program (Project SysteMTb HEALTH-F4-2010-241587). Research in the laboratory of P.S. is supported by Swiss National Science Foundation, EU NewTBVac (No. 241 745) and Tuberculosis Vaccine Initiative (TBVI).

\section{Supplementary data}

Supplementary data associated with this article can be found, in the online version, at http://dx.doi.org/10.1016/j.bmcl. 2014.04.004. These data include MOL files and InChiKeys of the most important compounds described in this article.

\section{References and notes}

1. Petrera, A.; Amstutz, B.; Gioia, M.; Hahnlein, J.; Baici, A.; Selchow, P.; Ferraris, D. M.; Rizzi, M.; Sbardella, D.; Marini, S.; Coletta, M.; Sander, P. Biol. Chem. 2012, 393, 631.

2. Ferraris, D. M.; Rizzi, M. Encyclopedia of Inorganic and Bioinorganic Chemistry In Scott, R. A., Ed.; John Wiley: Chichester, 2013. http://dx.doi.org/10.1002/ 9781119951438.eibc2171.

3. Master, S. S.; Rampini, S. K.; Davis, A. S.; Keller, C.; Ehlers, S.; Springer, B.; Timmins, G. S.; Sander, P.; Deretic, V. Cell Host Microbe 2008, 3, 224.

4. Chan, J.; Flynn J. Clin. Immunol. 2004, 110, 2.

5. Schroder, K.; Tschopp, J. Cell 2010, 140, 821.

6. Muttucumaru, D. G.; Smith, D. A.; McMinn, E. J.; Reese, V.; Coler, R. N.; Parish, T. Tuberculosis (Edinb.) 2011, 91, 111.

7. Johansen, P.; Fettelschoss, A.; Amstutz, B.; Selchow, P.; Waeckerle-Men, Y.; Keller, P.; Deretic, V.; Held, L.; Kundig, T. M.; Bottger, E. C.; Sander, P. Clin. Vaccine Immunol. 2011, 18, 907.

8. Kaufmann, S. H.; Gengenbacher, M. Curr. Opin. Biotechnol. 2012, 23, 900.

9. Ferraris, D. M.; Sbardella, D.; Petrera, A.; Marini, S.; Amstutz, B.; Coletta, M.; Sander, P.; Rizzi, M. J. Biol. Chem. 2011, 286, 32475.

10. Jones, G.; Willett, P.; Glen, R. C.; Leach, A. R.; Taylor, R. J. Mol. Biol. 1997, 267, 727.

11. Morris, G. M.; Huey, R.; Lindstrom, W.; Sanner, M. F.; Belew, R. K.; Goodsell, D. S.; Olson, A. J. J. Comput. Chem. 2009, 30, 2785.

12. Case, D. A.; Darden, T. A.; Cheatham, T.E.; Simmerling, C. L.; Wang, J.; Duke, R. E.; Luo, R.; Walker, R. C.; Zhang, W.; Merz, K. M.; Roberts, B.; Hayik, S.; Roitberg, A.; Seabra, G.; Swails, J.; Goetz, A. W.; Kolossváry, I.; Wong, K. F.; Paesani, F.; Vanicek, J.; Wolf, R. M.; Liu, J.; Wu, X.; Brozell, S. R.; Steinbrecher, T.; Gohlke, H.; Cai, Q.; Ye, X.; Wang, J.; Hsieh, M. J.; Cui, G.; Roe, D. R.; Mathews, D. H.; Seetin, M. G.; Salomon-Ferrer, R.; Sagui, C.; Babin, V.; Luchko, T.; Gusarov, S.; Kovalenko, A.; Kollman, P. A. AMBER 12, 2012. Available at: http:// ambermd.org/.

13. Mori, M.; Dietrich, U.; Manetti, F.; Botta, M. J. Chem. Inf. Model. 2010, 50, 638.

14. Mori, M.; Manetti, F.; Botta, M. J. Chem. Inf. Model. 2011, 51, 446.

15. Grant, J. A.; Gallardo, M. A.; Pickup, B. T. J. Comput. Chem. 1996, 17, 1653.

16. Kumar, B. R. P.; Basu, P.; Adhikary, L.; Nanjan, M. J. Synth. Commun. 2012, 42, 3089.

17. Survey on Scifinder (https://scifinder.cas.org/) and PubChem (https:// pubchem.ncbi.nlm.nih.gov/) was performed on 28th March 2014. Search of ZTB23 chemical structure on Scifinder provided only one match corresponding to compound ZTB23 without assigned stereochemistry (CAS number 30632421-0), including 11 commercial sources and a single literature reference (PCT WO 2009113795) where the synthesis of ZTB23 is not reported. Search on PubChem provided ZTB23 chemical structure without assigned stereochemistry and associated to a number of biological tests where the compound was inactive.

18. Altschul, S. F.; Gish, W.; Miller, W.; Myers, E. W.; Lipman, D. J. J. Mol. Biol. 1990, $215,403$. 